\title{
THE QUINTIC COMPLEX MOMENT PROBLEM
}

\author{
H. El-AZHar, A. HarRat, K. IDRissi AND E. H. ZEROUAli
}

\begin{abstract}
Let $\gamma^{(m)} \equiv\left\{\gamma_{i j}\right\}_{0 \leqslant i+j \leqslant m}$ be a given complex-valued sequence. The truncated complex moment problem (TCMP in short) involves determining necessary and sufficient conditions for the existence of a positive Borel measure $\mu$ on $\mathbb{C}$ such that $\gamma_{i j}=\int \bar{z}^{i} z^{j} d \mu$ for $0 \leqslant i+j \leqslant m$. The TCMP has been completely solved only when $m=1,2,3$ and 4 .

We provide in this paper a concrete solution to the, almost all, quintic TCMP (that is, when $m=5$ ). We also study the cardinality of the minimal representing measure. Based on the bi-variate recurrence sequences properties with some Curto-Fialkow's results. Our method intended to be useful for all odd-degree moment problems.
\end{abstract}

Mathematics subject classification (2010): 47A57, 15A83, 30E05, 44A60.

Keywords and phrases: Quintic complex moment problem, minimal representing measure, complexvalued bi-sequence.

\section{REFERENCES}

[1] N. I. AKHEIZER, The classical moment problem and some related questions in analysis, Oliver \& Boyd, 1965.

[2] C. Bayer And J. Teichmann, The proof of Tchakaloff's theorem, Proc. Amer. Math. Soc., 134 (10) (2006), 3035-3040.

[3] G. Blekherman, Nonnegative polynomials and sums of squares, J. Amer. Math. Soc. 25 (3) (2012), 617-635.

[4] R. E. CURTO AND L. A. FialKow, Recursively generated weighted shifts and the subnormal completion problem, Integral Equations Operator Theory 17 (2) (1993), 202-246.

[5] R. E. CURTO AND L. A. FIALKOW, Recursively generated weighted shifts and the subnormal completion problem II, Integral Equations Operator Theory 18 (4) (1994), 369-426.

[6] R. E. CURTO AND L. A. FialKow, Solution of the truncated complex moment problem for flat data, Volume 568, American Mathematical Soc., 1996.

[7] R. E. Curto And L. A. Fialkow, Flat Extensions of Positive Moment Matrices: Recursively Generated Relations, Volume 648, American Mathematical Soc., 1998.

[8] R. E. Curto AND L. A. Fialkow, Flat extensions of positive moment matrices: Relations in analytic or conjugate terms, In Nonselfadjoint operator algebras, operator theory, and related topics, pages 59-82. Springer, 1998.

[9] R. E. Curto AND L. A. Fialkow, Solution of the singular quartic moment problem, J. Operator Theory 48 (2) (2002), 315-354.

[10] R. E. CuRTO AND L. A. Fialkow, Truncated $K$-moment problems in several variables, J. Operator Theory 54 (1) (2005), 189-226.

[11] R. E. Curto, L. A. Fialkow, And H. M. MöLler, The extremal truncated moment problem, Integral Equations Operator Theory, 60 (2) (2008), 177-200.

[12] R. E. Curto And M. Putinar, Nearly subnormal operators and moment problems, J. Funct. Anal. 115 (2) (1993), 480-497.

[13] R. E. Curto And S. Yoo, Non-extremal sextic moment problems, J. Funct. Anal. 269 (3) (2015), $758-780$.

[14] R. E. CURTO AND S. Yoo, Concrete solution to the nonsingular quartic binary moment problem, Proc. Amer. Math. Soc. 144 (1) (2016), 249-258. 
[15] R. E. CURTO AND S. Yoo, The division algorithm in sextic truncated moment problems, Integral Equations Operator Theory 87 (4) (2017), 515-528.

[16] R. E. CURTO AND S. Yoo, A new approach to the nonsingular cubic binary moment problem, Ann. Funct. Anal. 9 (4) (2018), 525-536.

[17] O. Demanze, Moments and positivity, J. Math. Anal. Appl. 247 (2) (2000), 570-587.

[18] F. Dubeau, W. Motta, M. Rachidi, AND O. SAeKI, On weighted r-generalized Fibonacci sequences, The Fibonacci Quarterly 35 (2) (1997), 102-110.

[19] L. Fial Kow AND J. Nie, Positivity of riesz functionals and solutions of quadratic and quartic moment problems, J. Funct. Anal. 258 (1) (2010), 328-356.

[20] K. Idrissi And E. H. Zerouali, Charges solve the truncated complex moment problem, Infin. Dimens. Anal. Quantum Probab. Relat. Top. 21 (4) (2018), 1850027.

[21] K. IDRISSI AND E. H. ZerouAli, Multivariable recursively generated weighted shifts and the 2variable subnormal completion problem, Kyungpook Math. J., 58 (4) (2018), 711-732.

[22] D. P. Kimsey, Matrix-valued moment problems, PhD thesis, Drexel University, 2011.

[23] D. P. KiMSEY, The cubic complex moment problem, Integral Equations Operator Theory 80 (3) (2014), 353-378.

[24] A. LAMBert, A characterization of subnormal operators, Glasg. Math. J. 25 (1) (1984), 99-101.

[25] J. LASSERRE, Polynomials nonnegative on a grid and discrete optimization, Trans. Amer. Math. Soc., 354 (2) (2002) 631-649.

[26] J. B. LASSERRE, Global optimization with polynomials and the problem of moments, SIAM J. Optim. 11 (3) (2001), 796-817.

[27] J. B. Lasserre, Bounds on measures satisfying moment conditions, Ann. Appl. Probab.,12 (3) (2002), 1114-1137.

[28] J.-B. LASSERRE, Moments, positive polynomials and their applications, World Scientific, 2010.

[29] M. LAURENT, Sums of squares, moment matrices and optimization over polynomials, In Emerging applications of algebraic geometry, pages 157-270. Springer, 2009.

[30] M. PutinAR AND C. SCheiderer, Multivariate moment problems: geometry and indeterminateness, Ann. Sc. Norm. Super. Pisa Cl. Sci. (5) 5 (2) (2006), 137-158.

[31] D. Sarason, Moment problems and operators in Hilbert space, Moments in Mathematics, Proc. Sympos. Appl. Math. 37 (1987), 54-70.

[32] Y. L. SHMUL'YAN, An operator Hellinger integral (Russian), Mat. Sb. 91 (4) (1959), 381-430.

[33] J. A. Shohat And J. D. TAmarkin, The problem of moments, Math. Surveys I, Amer. Math. Soc., Providence, RI, 1943.

[34] J. STOCHEL, Solving the truncated moment problem solves the full moment problem, Glasg. Math. J. 34 (3) (2001), 335-341.

[35] V. TCHAKALOFF, Formules de cubatures mécaniques à coefficients non négatifs, Bull. Sci. Math. 81 (2) (1957), 123-134.

[36] F.-H. VAsilescu, Dimensional stability in truncated moment problems, J. Math. Anal. Appl., 388 (1) (2012), 219-230.

[37] S. Yoo, Extremal sextic truncated moment problems, $\mathrm{PhD}$ thesis, The University of Iowa, 2010.

[38] S. Yoo, Sextic moment problems on 3 parallel lines, Bull. Korean Math. Soc., 54 (1) (2017), 299-318. 